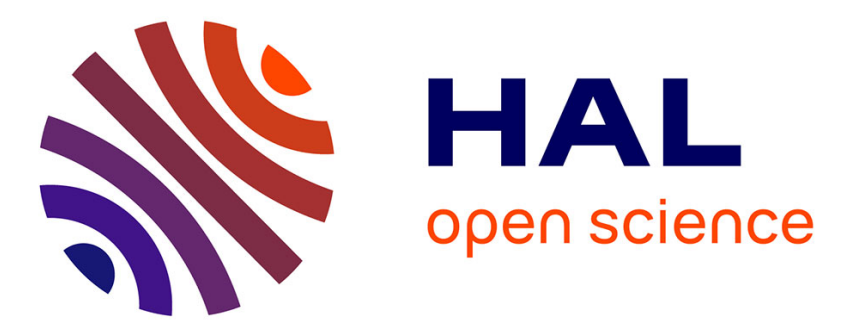

\title{
Structural properties of various sodium thiogermanate glasses through DFT-based molecular dynamics simulations
}

Sebastien Blaineau, Philippe Jund

\section{- To cite this version:}

Sebastien Blaineau, Philippe Jund. Structural properties of various sodium thiogermanate glasses through DFT-based molecular dynamics simulations. Physical Review B: Condensed Matter and Materials Physics (1998-2015), 2006, pp.054203. 10.1103/PhysRevB.74.054203 . hal-00583914

\section{HAL Id: hal-00583914 https://hal.science/hal-00583914}

Submitted on 7 Apr 2011

HAL is a multi-disciplinary open access archive for the deposit and dissemination of scientific research documents, whether they are published or not. The documents may come from teaching and research institutions in France or abroad, or from public or private research centers.
L'archive ouverte pluridisciplinaire HAL, est destinée au dépôt et à la diffusion de documents scientifiques de niveau recherche, publiés ou non, émanant des établissements d'enseignement et de recherche français ou étrangers, des laboratoires publics ou privés. 


\title{
Structural properties of various sodium thiogermanate glasses through DFT-based molecular dynamics simulations
}

\author{
Sébastien Blaineau and Philippe Jund \\ Laboratoire de Physicochimie de la Matière Condensée, Université Montpellier 2, Place E. Bataillon, Case 03, 34095 Montpellier, \\ France \\ (Received 9 January 2006; revised manuscript received 11 May 2006; published 7 August 2006)
}

\begin{abstract}
We present a study of the structural properties of $(x) \mathrm{Na}_{2} \mathrm{~S}-(1-x) \mathrm{GeS}_{2}$ glasses through density-functionaltheory-based molecular dynamics simulations, at different sodium concentrations $(0<x<0.5)$. We computed the radial pair correlation functions as well as the total and partial structure factors. We also analyzed the evolution of the corner- and edge-sharing intertetrahedral links with the sodium concentration and show that the sodium ions exclusively destroy the former. With the increase of the sodium concentration the "standard" first sharp diffraction peak disappears and a new prepeak appears in the structure factor which can be traced back in the Na-Na partial structure factor. This self-organization of the sodium ions is coherent with Na-rich zones that we find at high modifier concentration.
\end{abstract}

DOI: 10.1103/PhysRevB.74.054203

PACS number(s): 61.43.Bn, 61.43.Fs, 71.15.Pd, 63.50.+x

\section{INTRODUCTION}

Amorphous $(x) \mathrm{Na}_{2} \mathrm{~S}-(1-x) \mathrm{GeS}_{2}$ systems are interesting glasses, presenting a high ionic conductivity at room temperature. These materials can be used as model systems for efficient solid electrolytes even though practical applications are more often based on glasses containing lithium or silver ions, for example. Although the ionic conduction process has been clearly observed in sodium thiogermanate glasses, its microscopic origin is still not clearly understood. As in many other glassy systems, the atomic mechanisms responsible for these transport properties have not yet been clearly determined, which explains the large number of studies dedicated to this topic in the past few years. ${ }^{1}$ In particular, it is interesting to determine if the modifier ions follow preferential pathways inside the amorphous system, ${ }^{2}$ and if this process is dependent on the glass type. To that end, theoretical studies such as molecular dynamics simulations can provide detailed information concerning the microscopic properties of these chalcogenide glasses.

In previous studies we have analyzed the physical and chemical properties of $\mathrm{GeS}_{2}$ glasses via density-functionaltheory- (DFT-)based molecular dynamics simulations. ${ }^{3-5}$ The results obtained from our model were in very good agreement with the available experimental data. Subsequently we studied the vibrational and electronic properties of sodium thiogermanate (Na-Ge-S) glasses using the same description. ${ }^{6}$ In the present work, we focus on the structural properties of $(x) \mathrm{Na}_{2} \mathrm{~S}-(1-x) \mathrm{GeS}_{2}$ amorphous systems using the same DFT-based model in order to determine the impact of the $\mathrm{Na}^{+}$ions on the structure of germanium disulfide glasses. It should be noted that there is a lack of experimental results in the literature concerning these doped chalcogenide glasses. Thus no extensive comparison with experiments is possible at this time, and our results should be considered as a first step in the study of the structural properties of $(x) \mathrm{Na}_{2} \mathrm{~S}-(1-x) \mathrm{GeS}_{2}$ glasses.

This paper is organized as follows. Section II is dedicated to the description of the theoretical foundations of our model. Subsequently, we present the results obtained in our simulations in Sec. III. Finally, Sec. IV contains the major conclusions of our work.

\section{MODEL}

The mathematical model we have used was developed by Sankey and Niklewski. ${ }^{7}$ It is included in a first-principlestype molecular dynamics code, called FIREBALL96, that is based on the $\mathrm{DFT}^{8}$ within the local density approximation. ${ }^{9,10}$ A tight-binding-like linear combination of pseudoatomic orbitals, satisfying the atomic self-consistent Hohenberg-Kohn-Sham equations, ${ }^{11}$ is used to determine the electronic eigenstates of the system. A basis set of one $s$ and three $p$ pseudo-orbitals, slightly excited since they vanish outside a cutoff radius of $5 a_{o}(2.645 \AA)$, is required.

The pseudopotential approximation is used to replace the core electrons with an effective potential which acts on the valence electrons, and Hamman-Schluter-Chiang pseudopotentials were used in this work. ${ }^{12}$ The Hamiltonian is calculated through the use of the Harris functional, ${ }^{13}$ which avoids long self-consistent calculations by a zeroth-order approximation of the self-consistent density. Further details on the method can be found in the original paper. ${ }^{14}$ Periodic boundary conditions are applied in order to limit surface effects, and only the $\Gamma$ point is used to sample the Brillouin zone. This model has given excellent results in several different chalcogenide systems over the past few years. ${ }^{3,15,16}$

In the present work we computationally melted a crystalline $\alpha$-GeS $\mathrm{S}_{2}$ configuration containing 258 particles at $2000 \mathrm{~K}$ during $60 \mathrm{ps}$ (24 000 time steps) until we obtained an equilibrated liquid. Subsequently we randomly replaced $\mathrm{GeS}_{4}$ tetrahedral units by $\mathrm{Na}_{2} \mathrm{~S}_{3}$ units, in order to obtain a given concentration of modifier without affecting the global electrical neutrality of the system. This procedure has been used in a similar manner in $\mathrm{SiO}_{2}$ glasses, ${ }^{17}$ and keeps the total number of atoms, $N$, unchanged (258 particles in this work).

Following this scheme, we generated eight $(x) \mathrm{Na}_{2} \mathrm{~S}-(1$ $-x) \mathrm{GeS}_{2}$ samples with different sodium concentrations $(x$ $=0,0.015,0.03,0.06,0.11,0.2,0.33$, and 0.5). The bounding 


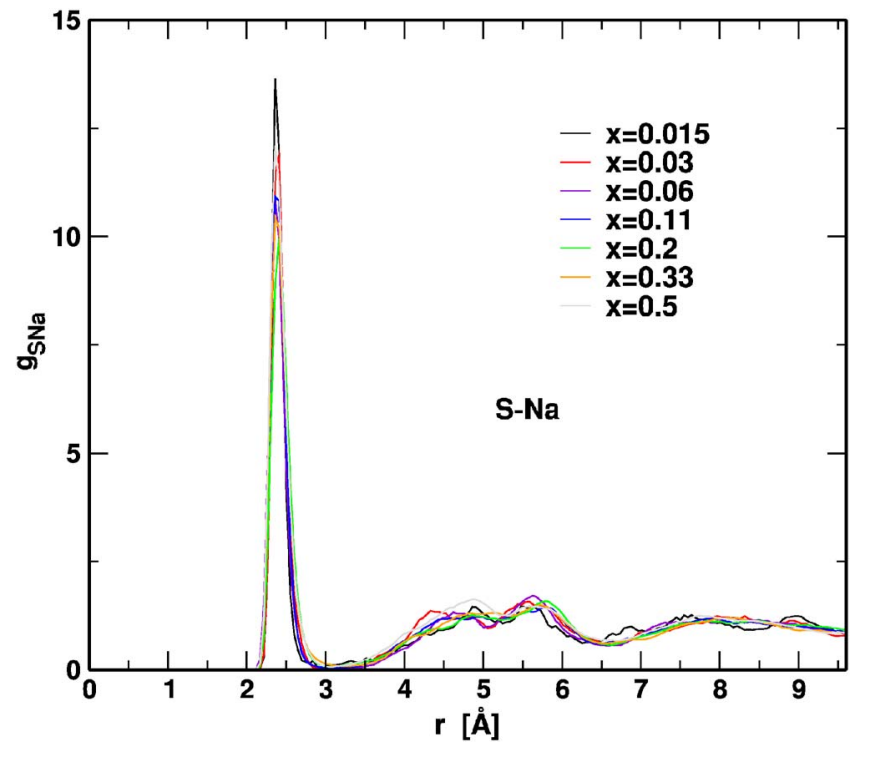

FIG. 1. (Color online) Radial S-Na pair distribution functions of $(x) \mathrm{Na}_{2} \mathrm{~S}-(1-x) \mathrm{GeS}_{2}$ glasses for $0<x \leq 0.5$.

box was rescaled for each sample so that the density matched its experimental counterpart [with cell lengths ranging from $19.21 \AA$ for $x=0$ (Ref. 18) to $18.3 \AA$ for $x=0.5$ (Ref. 19)]. Since the experimental densities are not exactly the equilibrium densities of the model, the pressure inside the samples is not exactly equal to zero. Nevertheless it is extremely small $(<0.4 \mathrm{GPa})$, demonstrating the quality of the model. The resulting system was subsequently melted at $2000 \mathrm{~K}$ during $60 \mathrm{ps}$ in order for the system to lose memory of the previous artificial configuration. When the liquid reached an equilibrium state, the systems were quenched at a quenching rate of $6.8 \times 10^{14} \mathrm{~K} / \mathrm{s}$, decreasing the temperature to $300 \mathrm{~K}$, passing through the glass transition temperature $T_{g}$. The samples were then relaxed at $300 \mathrm{~K}$ over a period of $100 \mathrm{ps}$. In order to improve the statistics of our results, five samples, starting from independent liquid configurations, were generated for each concentration. The results presented here have been averaged over these five samples.

\section{RESULTS}

In a previous work, ${ }^{5}$ we determined that the atomic charges in $\mathrm{GeS}_{2}$ glasses using a Löwdin description ${ }^{20}$ are equal to +0.94 for $\mathrm{Ge}$ atoms and -0.47 for the $\mathrm{S}$ particles. Even though homopolar bonds exist in this glass, ${ }^{4}$ no $\mathrm{Ge}$ -Na bonds were seen in our samples at the concentrations studied here $(0<x \leq 0.5)$. Therefore we can study the impact of the sodium ions on the short-range order of germanium disulfide glasses by analysis of the S-Na bonds exclusively.

Among the radial pair correlation functions $g_{\alpha \beta}(r)$ defined as

$$
g_{\alpha \beta}(r)=\frac{V}{4 \pi r^{2} N_{\alpha} d r} d n_{\beta}
$$

for the different $(\alpha, \beta)$ pairs, we can hence concentrate on the $g_{\mathrm{SNa}}(r)$ function. It can be seen in Fig. 1 that the $g_{\mathrm{SNa}}(r)$

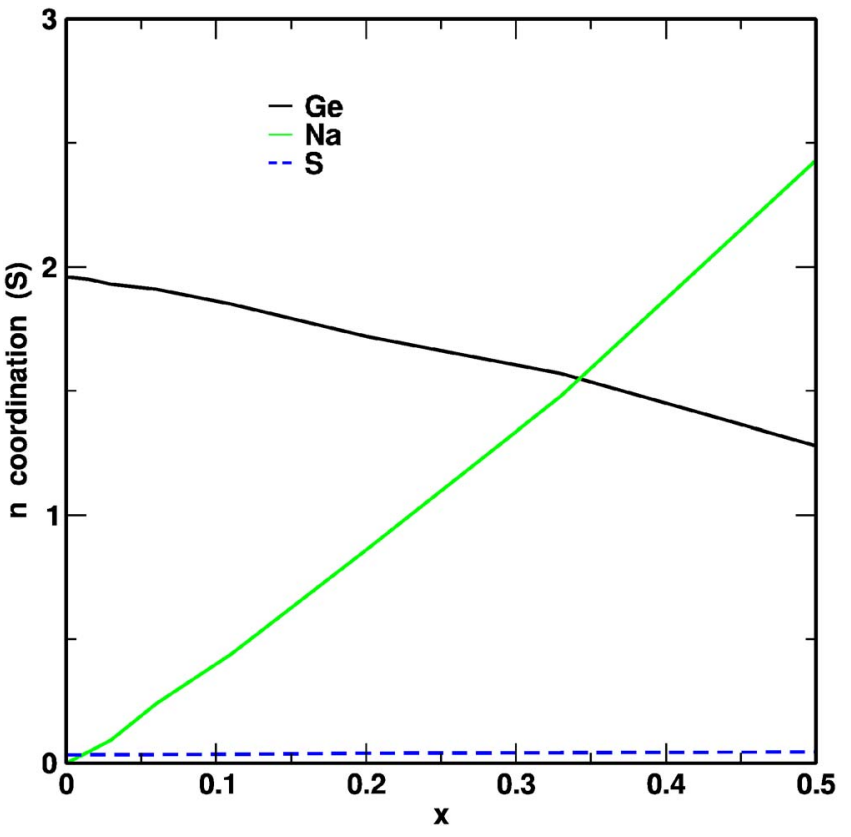

FIG. 2. (Color online) Coordination number of sulfur atoms in sodium thiogermanate samples, plotted against $x$, the concentration of sodium atoms.

for S-Na pairs does not change significantly with the concentration of the sodium modifier. The first-neighbor peak appears at $2.4 \AA$ for all concentrations $(0<x \leq 0.5)$, which is slightly inferior to experimental data ${ }^{21}$ concerning crystalline sodium thiogermanate systems $(2.71 \AA)$. However we tested several glassy samples with the program ${ }^{22}$ SIESTA in a selfconsistent $a b$ initio description, using the most accurate basis set available in the code for the description of the atomic orbitals (double zeta polarized + generalized gradient approximation): the results were very similar $\left(d_{\mathrm{S}-\mathrm{Na}}=2.45 \AA\right)$ to those obtained with FIREBALL96.

The different coordination numbers $n_{\alpha \beta}$ can also be calculated. They correspond to the average number of $\beta$ neighbors of a given $\alpha$ atom within a sphere of radius $r_{\text {min }}, r_{\text {min }}$ being the first minimum of the corresponding $g_{\alpha \beta}(r)$. We show in Fig. 2 the evolution of the coordination number of the sulfur atoms with the three elements present in our system. It can be seen that the value of $n_{\mathrm{S}-\mathrm{Ge}}$ decreases with the addition of sodium modifier from 1.95 for $x=0$ to 1.3 for $x$ $=0.5$. This indicates that the $\mathrm{Na}^{+}$ions break several intertetrahedral connections in order to be connected to the sulfur atoms, which explains the increasing value of $n_{\mathrm{S}-\mathrm{Na}}(2.45$ for $x=0.5)$. The value of $n_{\mathrm{S}-\mathrm{S}}$ relative to homopolar bonds remains constant without any dependency on the sodium concentration. Finally, the average coordination number of the sodium atoms relative to the sulfur atoms, $n_{\mathrm{Na}-\mathrm{S}}$, has been found close to 3.27 for $x=0.5$. In fact, the $\mathrm{Na}^{+}$particles in the system are connected to three or four sulfur atoms, which is consistent with the results obtained by Foix et al.. in cluster simulations of $\mathrm{Na}_{2} \mathrm{GeS}_{3}$ glasses using a Hartree-Fock model. ${ }^{21}$

We reported in a previous study ${ }^{4}$ that $14.53 \%$ of the sulfur atoms in glassy $\mathrm{GeS}_{2}$ systems are nonbridging (i.e., connected to only one Ge atom). These bond defects have been 


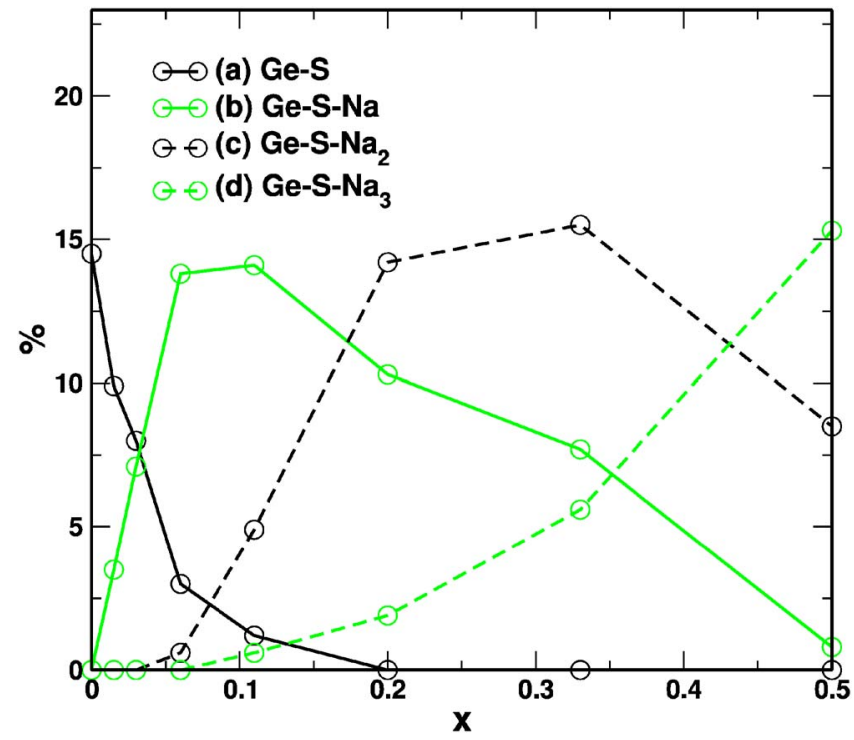

FIG. 3. (Color online) Proportion of nonbridging sulfur atoms in $(x) \mathrm{Na}_{2} \mathrm{~S}-(1-x) \mathrm{GeS}_{2}$ glasses. We distinguish the $\mathrm{S}$ atoms connected to (a) zero, (b) one, (c) two, and (d) three $\mathrm{Na}^{+}$ions.

found ${ }^{5}$ to create a negatively charged electronic environment around the terminal sulfur. Therefore it is interesting to study the evolution of these nonbridging sulfur particles with the introduction of $\mathrm{Na}^{+}$cations. We distinguish here between the terminal sulfur atoms connected to (a) zero, (b) one, (c) two, or (d) three sodium ions, and represent in Fig. 3 the evolution of these structural entities in $(x) \mathrm{Na}_{2} \mathrm{~S}-(1-x) \mathrm{GeS}_{2}$ for $0 \leq x$ $\leq 0.5$.

One can see that the proportion of terminal sulfur atoms that are not linked to a sodium ion decreases significantly as soon as $x$ is nonzero. This variation is counterbalanced by the increase of the proportion of terminal sulfur atoms connected to one sodium atom, which reaches approximately the same value of $\approx 15 \%$ of the $\mathrm{S}$ particles in the sample. We can therefore deduce that, as expected, the nonbridging sulfurs present in $g-\mathrm{GeS}_{2}$ significantly attract the $\mathrm{Na}^{+}$ions. Between $x=0.11$ and 0.33 these structural entities decrease [Fig. 3(b)], and are counterbalanced by the increase of terminal sulfur connected to two Na ions [Fig. 3(c)], which also reaches the value of $\approx 15 \%$. Finally, for $x>0.33$ the proportion of terminal sulfurs connected to three sodium ions reaches $\approx 15 \%$ as in the aforementioned cases. This shows that the nonbridging sulfur atoms observed in $g-\mathrm{GeS}_{2}$ attract several $\mathrm{Na}^{+}$ions in sodium thiogermanate glasses.

The study of the short-range charge deviation in $g-\mathrm{GeS}_{2}$ has also revealed the existence of "positively" charged zones, which are mainly caused by threefold-coordinated sulfur and homopolar bonds. ${ }^{5}$ We find that these positively charged zones remain basically unchanged in $(x) \mathrm{Na}_{2} \mathrm{~S}-(1$ $-x) \mathrm{GeS}_{2}$ glasses, and that the $\mathrm{Na}^{+}$ions never connect to threefold-coordinated sulfur atoms. Hence negatively and positively charged zones in $g-\mathrm{GeS}_{2}$ appear to attract and repel the $\mathrm{Na}^{+}$cations, respectively. This observation, which seems to be self-evident, is in fact relevant since all the different samples have been melted, quenched, and relaxed independently. Despite that, we find similarly charged zones in

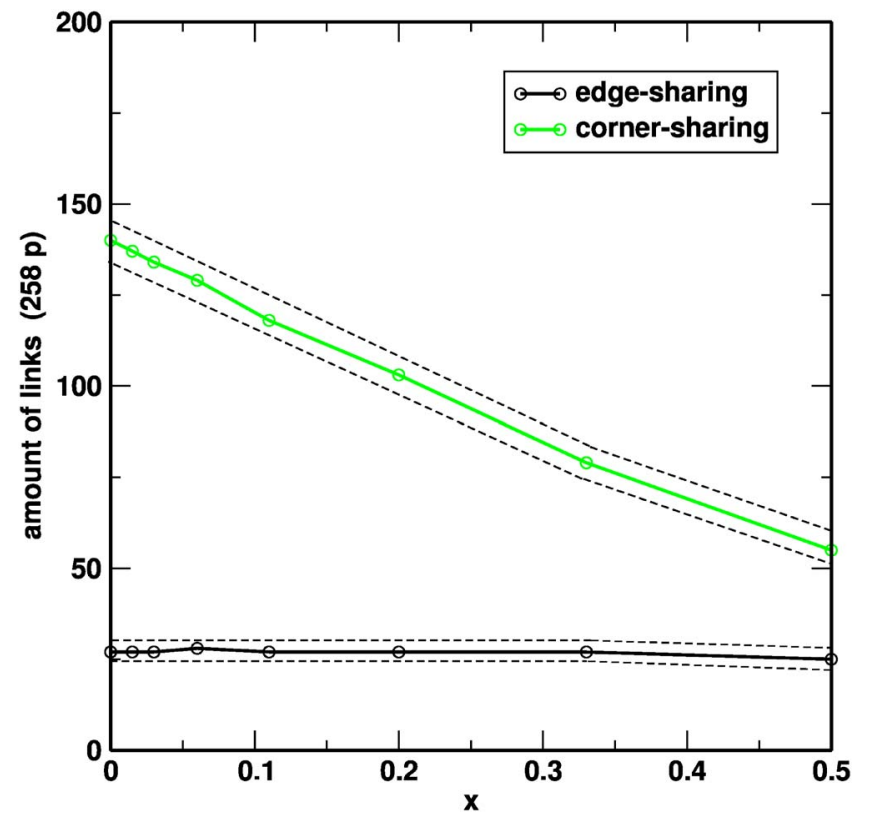

FIG. 4. (Color online) Number of edge-sharing and cornersharing links in $(x) \mathrm{Na}_{2} \mathrm{~S}-(1-x) \mathrm{GeS}_{2}$ glasses, plotted against $x$, the concentration of sodium atoms.

comparable proportions in all of these samples.

The decrease of the $n_{\mathrm{S} \text {-Ge }}$ coordination number with increasing sodium content observed in Fig. 2 shows that an increasing number of intertetrahedral bonds are destroyed by the addition of sodium ions. Since two different types of intertetrahedral links exist in glassy $\mathrm{GeS}_{2}$ (edge-sharing and corner-sharing links), it is interesting to determine which type of S-Ge connections are destroyed by the introduction of the $\mathrm{Na}^{+}$ions. These two types of intertetrahedral connections can be distinguished in the radial pair correlation function of germanium-germanium pairs, ${ }^{4}$ leading to $\mathrm{Ge}-\mathrm{Ge}$ distances equal to $2.91 \AA$ (edge sharing) and $3.41 \AA$ (corner sharing). In Fig. 4 we present the total number of cornersharing and edge-sharing links in the different samples as a function of sodium concentration. It can clearly be seen that exclusively corner-sharing links are destroyed by the introduction of sodium cations whereas the number of edgesharing links remains constant for all sodium concentrations. This result is not predictable a priori since very different results may be obtained depending on the glass and the modifier. For example, experimental studies on chalcogenide $\mathrm{SiS}_{2}$ glasses have shown that $\mathrm{Li}^{+}$cations destroy exclusively edge-sharing links, ${ }^{23,24}$ while the addition of $\mathrm{Na}^{+}$ions was found to break corner-sharing and edge-sharing links in equal proportions. ${ }^{25}$ Here we find that sodium ions destroy exclusively corner-sharing links in $\mathrm{GeS}_{2}$ glasses but this result still has to be confirmed (or refuted) experimentally.

In order to analyze the global impact of the sodium ions on the structure of thiogermanate glasses, we studied next the total static structure factor $S(q)$ of our $(x) \mathrm{Na}_{2} \mathrm{~S}-(1$ $-x) \mathrm{GeS}_{2}$ glasses. The total static structure factor was computed using the following formulas: 


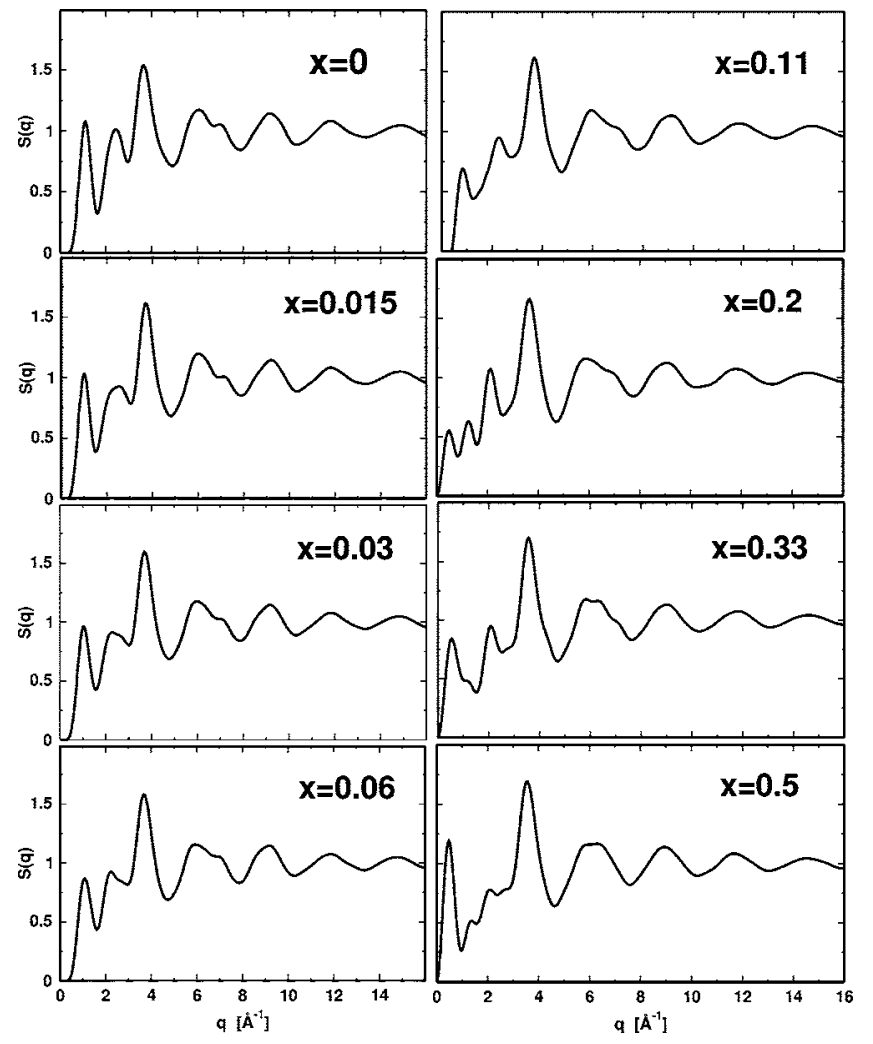

FIG. 5. The simulated static structure factors of the different sodium thiogermanate glasses.

$$
S(q)=1+4 \pi \frac{N}{V} \int_{0}^{r_{\max }}[g(r)-1] \frac{\sin (q r)}{q r} r^{2} d r
$$

where

$$
g(r)=\frac{\left(\sum_{\alpha, \beta} c_{\alpha} b_{\alpha} c_{\beta} b_{\beta} g_{\alpha \beta}(r)\right)}{\left(\sum_{\alpha} c_{\alpha} b_{\alpha}\right)^{2}}
$$

In the above equations, $r_{\max }=L / 2$ where $L$ is the edge of the box, the $g_{\alpha \beta}(r)$ are the radial pair correlation functions $(\alpha, \beta=\mathrm{Ge}, \mathrm{S}, \mathrm{Na}), c_{\alpha}$ and $c_{\beta}$ are the concentrations of the species $\alpha$ and $\beta$, and $b_{\alpha}$ and $b_{\beta}$ are their scattering lengths taken equal to $8.18 \mathrm{fm}$ for $\mathrm{Ge}, 2.84 \mathrm{fm}$ for $\mathrm{S}$, and $3.63 \mathrm{fm}$ for $\mathrm{Na}^{26}$ The results for $S(q)$, calculated using the above definition, are shown in Fig. 5 for the different values of $x$ (even though these results are directly comparable to neutron scattering experiments, no experimental data are available to our knowledge).

The first sharp diffraction peak (FSDP), which is a signature of amorphous materials, appears at $q=1 \AA^{-1}$ in $g$ - $\mathrm{GeS}_{2}{ }^{3}$ This peak reveals the existence of a structural order on a length scale $d$ corresponding to $d=2 \pi / q \approx 6.3 \AA$ in germanium disulfide glasses. In sodium thiogermanate glasses, it can be seen that the intensity of the FSDP decreases significantly with $x$ for $x \leq 0.2$. This decrease of the FSDP has also been observed experimentally in silver thiogermanate glasses. ${ }^{24}$ This means that the introduction of ions disturbs

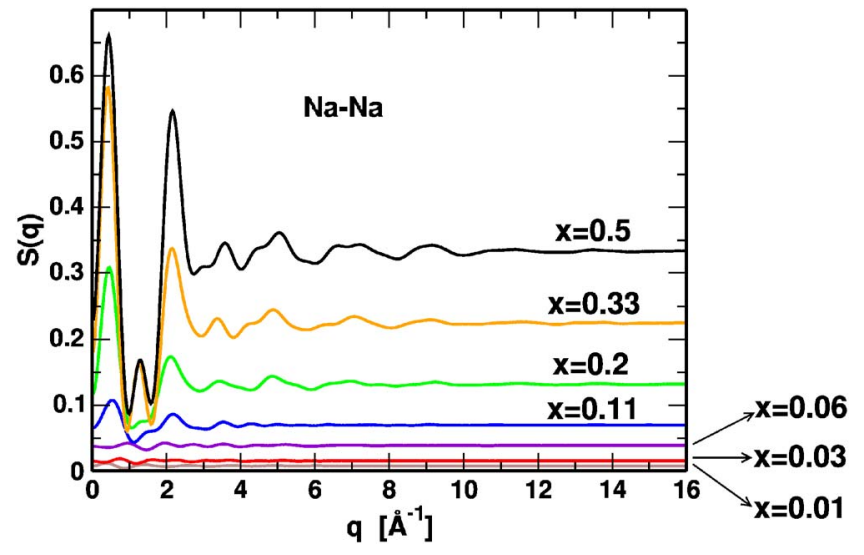

FIG. 6. (Color online) Partial $S(q)$ for Na-Na pairs at different sodium concentrations versus $q$.

the intermediate-range order of the glassy matrix by breaking corner-sharing intertetrahedral connections. However for $x$ $\geq 0.2$, a new feature appears in the structure factor at a lower value of $q\left(q=0.5 \AA^{-1}\right)$. For $x=0.5$ this prepeak reaches almost the same intensity as the original FSDP, which almost completely vanishes and shifts to a slightly higher $q$ value. In order to find the origin of this new peak we calculated the partial structure factors [computed from the radial pair correlation functions $\left.g_{\alpha \beta}(r)\right]$ in the different samples, and in particular the Na-Na partial structure factor (Fig. 6). We find that the sodium-sodium pairs are mainly responsible for the prepeak observed in Fig. 5. It can indeed be seen that a peak emerges at $q=0.5 \AA^{-1}$ for $x \geq 0.11$ and subsequently grows with increasing $x$. To analyze the structural arrangement of the sodium atoms, which are obviously not distributed homogeneously inside the simulation box, we present the density of sodium atoms in $(0.33) \mathrm{Na}_{2} \mathrm{~S}-(0.66) \mathrm{GeS}_{2}$ averaged over the relaxation time in Fig. 7. To that end, we divide the bounding box into $1000(10 \times 10 \times 10)$ elementary volume elements. We subsequently calculated the number of $\mathrm{Na}$ atoms located in each specific volume element, and integrated this quantity over the relaxation time. Hence each sphere represented in Fig. 7 is located at the center of a volume element, and its size increases [becomes darker (more red in color)] with the local $\mathrm{Na}$ density (the largest sphere and thus the highest density corresponds to $0.154 \AA^{-3}$ ). This figure shows that our sodium thiogermanate sample contains several zones which have a high concentration of $\mathrm{Na}^{+}$and other zones which have a poor concentration of $\mathrm{Na}^{+}$ions. When we analyzed the local structure around the sodium atoms we found that most of them are in a $\mathrm{Na}_{2} \mathrm{~S}$-like environment, since for $x=0.5,23.5 \%$ of all the sulfur atoms are exclusively connected to $\mathrm{Na}$ ions, i.e., without any germanium atoms as their nearest neighbors. These sodium-rich zones could be the static "remainders" of conduction channels that could be observed at higher temperatures, similarly to what has been reported in sodium-enriched silicate glasses via classical molecular dynamics simulations and inelastic neutron scattering experiments. ${ }^{17,27}$ If we accept this hypothesis, then, since the new prepeak appears only for high sodium concentrations (see Fig. 6), conduction channels would be absent at low sodium content and this could explain the experimentally 


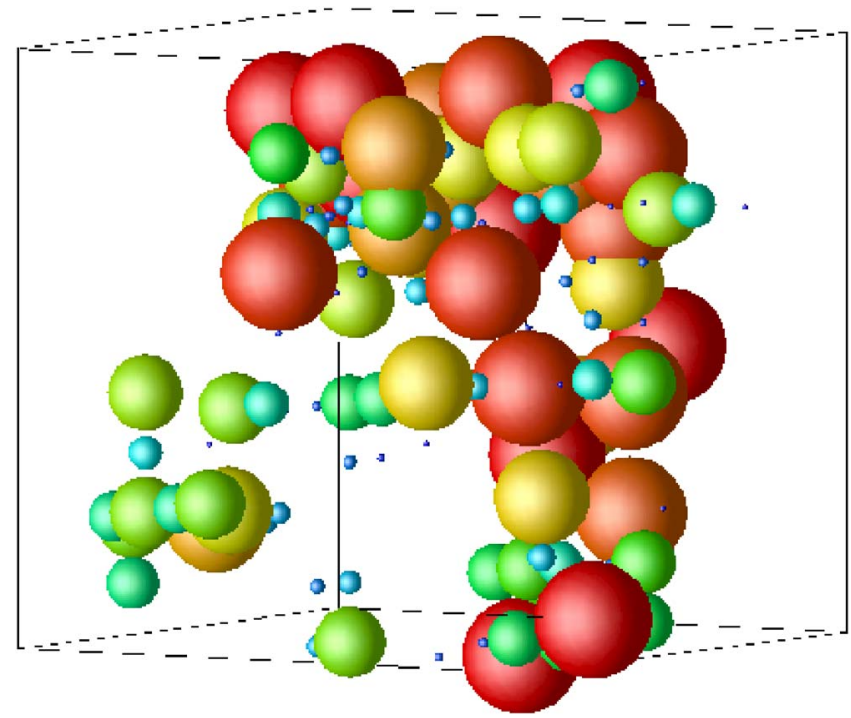

FIG. 7. (Color online) Illustration of the local Na density in a (0.33) $\mathrm{Na}_{2}-(0.66) \mathrm{GeS}_{2}$ sample integrated over the relaxation time (100 ps). The largest sphere corresponds to a $\mathrm{Na}$ density of $0.154 \AA^{-3}$.

observed increase in the sodium diffusion constant when the sodium concentration is greater than $x=0.10 .^{28}$ Unfortunately, the DFT-based model that we used to perform these simulations is too computationally expensive, making it difficult to reach the simulation time required to study the diffusion of the $\mathrm{Na}^{+}$ions, and to analyze the evolution of these zones from a dynamical point of view. This is the reason why the sodium-rich zones presented in Fig. 7 cannot be directly assimilated into the diffusion channels.

Most of these results have no experimental counterparts and therefore need still to be confirmed or invalidated, but neutron diffraction studies on these systems have been performed and the results should be available in the not so distant future.

To pursue this study further it would also be interesting to analyze the influence of the nature of the modifier on the results. Thus similar studies on silver thiogermanate glasses are currently in progress.

\section{CONCLUSION}

We have studied the structural properties of sodium thiogermanate glasses via DFT-based molecular dynamics simulations for eight different modifier concentrations. We find that the structure of these chalcogenide glasses is significantly influenced by the extended positively and negatively charged zones observed in amorphous $\mathrm{GeS}_{2} \cdot{ }^{5}$ As expected, the sodium cations are mainly attracted by the negative zones and avoid the positive regions. At high sodium concentrations, the $\mathrm{Na}^{+}$ions therefore become localized in space, and zones containing high and low density of sodium cations can be observed. These $\mathrm{Na}_{2} \mathrm{~S}$-rich zones could be the low-temperature analogs of the conduction channels observed in silicate glasses, where the diffusing ions follow preferential pathways. We have also determined that the addition of sodium cations inside the $(x) \mathrm{Na}_{2} \mathrm{~S}-(1$ $-x) \mathrm{GeS}_{2}$ system destroys exclusively the corner-sharing links for $x \leq 0.5$.

The structure factor of the different samples shows that the FSDP that appears in amorphous $\mathrm{GeS}_{2}$ at $q=1 \AA^{-1}$ decreases significantly with the addition of sodium ions. At high sodium concentrations $(x \geq 0.11)$, a prepeak appears at $q=0.5 \AA^{-1}$, which corresponds to a distance in real space of $12.6 \AA$. The Na-Na partial $S(q)$ shows that the Na-Na pairs are responsible for this prepeak indicating a nonhomogeneous distribution of the $\mathrm{Na}^{+}$ions inside the glass, which is consistent with the existence of the aforementioned sodiumrich zones.

\section{ACKNOWLEDGMENT}

Parts of the simulations have been performed on the computers of the "Centre Informatique National de l'Enseignement Supérieur” (CINES) in Montpellier.
${ }^{1}$ See, for example, Atomic Structure and Transport in Glassy Materials, special issue of J. Phys.: Condens. Matter 15, S1509 (2003).

${ }^{2}$ G. N. Greaves, J. Non-Cryst. Solids 71, 203 (1985); Miner. Mag. 64, 441 (2000), and references therein.

${ }^{3}$ S. Blaineau, P. Jund, and D. A. Drabold, Phys. Rev. B 67, 094204 (2003).

${ }^{4}$ S. Blaineau and P. Jund, Phys. Rev. B 69, 064201 (2004).

${ }^{5}$ S. Blaineau and P. Jund, Phys. Rev. B 70, 184210 (2004).

${ }^{6} \mathrm{~S}$. Blaineau and P. Jund, in Combining Simulations and Neutron Scattering Experiments: From Models to Systems of Biological and Technological Interest, special issue of Chem. Phys. 317, 137 (2005).

${ }^{7}$ O. F. Sankey and D. J. Niklewski, Phys. Rev. B 40, 3979 (1989).

${ }^{8}$ P. Hohenberg and W. Kohn, Phys. Rev. 136, B864 (1964).

${ }^{9}$ D. M. Ceperley and B. J. Alder, Phys. Rev. Lett. 45, 566 (1980).
${ }^{10}$ J. P. Perdew and A. Zunger, Phys. Rev. B 23, 5048 (1981).

${ }^{11}$ W. Kohn and L. J. Sham, Phys. Rev. 140, A1133 (1965).

${ }^{12}$ G. B. Bachelet, D. R. Hamann, and M. Schluter, Phys. Rev. B 26, 4199 (1982).

${ }^{13}$ J. Harris, Phys. Rev. B 31, 1770 (1985).

${ }^{14}$ A. A. Demkov, J. Ortega, O. F. Sankey, and M. P. Grumbach, Phys. Rev. B 52, 1618 (1995).

${ }^{15}$ M. Durandurdu, D. A. Drabold, and N. Mousseau, Phys. Rev. B 62, 15307 (2000).

${ }^{16}$ J. Li and D. A. Drabold, Phys. Rev. B 64, 104206 (2001).

${ }^{17}$ P. Jund, W. Kob, and R. Jullien, Phys. Rev. B 64, 134303 (2001).

${ }^{18}$ P. Boolchand, J. Grothaus, M. Tenhover, M. A. Hazle, and R. K. Grasselli, Phys. Rev. B 33, 5421 (1986).

${ }^{19}$ J. Olivier-Fourcade, J. C. Jumas, M. Ribes, E. Philippot, and M. Maurin, J. Solid State Chem. 23, 155 (1978).

${ }^{20}$ P.-O. Lowdin, J. Chem. Phys. 18, 365 (1950). 
${ }^{21}$ D. Foix, Ph.D. thesis, Université de Pau, France, 2003.

${ }^{22}$ http://www.uam.es/departamentos/ciencias/fismateriac/siesta/

${ }^{23}$ M. Micoulaut and R. Kerner, J. Phys.: Condens. Matter 9, 2551 (1997).

${ }^{24}$ J. H. Lee, A. P. Owens, A. Pradel, A. C. Hannon, M. Ribes, and S. R. Elliott, Phys. Rev. B 54, 3895 (1996).
${ }^{25}$ A. Pradel, G. Taillades, M. Ribes, and H. Eckert, J. Non-Cryst. Solids 188, 75 (1995).

${ }^{26}$ http://www.ncnr.nist.gov/resources/n-lengths/

${ }^{27}$ A. Meyer, J. Horbach, W. Kob, F. Kargl, and H. Schober, Phys. Rev. Lett. 93, 027801 (2004).

${ }^{28}$ A. Pradel, N. Kuvata, and M. Ribes (unpublished). 